\title{
BMJ Open Patient factors driving overuse of cardiac catheterisation: a qualitative study with 25 participants from two German teaching practices
}

\author{
Anna Herwig, ${ }^{1}$ Dorothea Dehnen, ${ }^{1}$ Birgitta Weltermann ${ }^{1,2}$
}

To cite: Herwig A, Dehnen D, Weltermann B. Patient factors driving overuse of cardiac catheterisation: a qualitative study with 25 participants from two German teaching practices. BMJ Open 2019;9:e024600. doi:10.1136/ bmjopen-2018-024600

- Prepublication history and additional material for this paper are available online. To view these files, please visit the journal online (http://dx.doi org/10.1136/bmjopen-2018024600)

Received 4 June 2018 Revised 20 February 2019 Accepted 25 February 2019

\section{Check for updates}

(c) Author(s) (or their employer(s)) 2019. Re-use permitted under CC BY-NC. No commercial re-use. See rights and permissions. Published by BMJ.

${ }^{1}$ Institute for General Medicine, University Hospital Essen, Essen, Germany

${ }^{2}$ Institute for General Practice and Family Medicine, University Hospital Bonn, Bonn, Germany

Correspondence to

Anna Herwig;

anna.herwig@uk-essen.de

\section{ABSTRACT}

Objectives Percutaneous coronary interventions do not provide a benefit over medical therapy for stable patients. However, an overuse of cardiac catheterisation (CC) for stable coronary artery disease (CAD) is documented in Germany and other countries. In this study, we aim to understand patient factors that foster this overuse. Design Our study is an exploratory qualitative interview study with narrative, structured interviews. The interviews were analysed using qualitative content analysis by Mayring.

Setting The interviews were conducted in two German teaching practices.

Participants 24 interviews with 25 patients were conducted; 17 (68\%) patients were male, the average age was 73.9 years (range 53-88 years). All patients suffered from CAD and had undergone at least one CC. Patients with known anxiety disorders were excluded from the study.

Results The analysis identified six patient factors which contributed to or prevented the overuse of CC: (1) unquestioned acceptance of prescheduled appointments for procedures/convenience; (2) disinterest in and/or lack of disease-specific knowledge; (3) helplessness in situations with varying opinions on the required care; (4) fear of another cardiac event, (5) patient-physician relationship and (6) the patient's experience that repeat interventions did not result in a change of health status or care.

Conclusions Conducted in a country with documented overuse of CC, we showed that most patients trusted their physicians' recommendations for repeat coronary angiographies even if they were asymptomatic. Strategies to align physician adherence with guidelines and corresponding patient information are needed to prevent overuse.

\section{INTRODUCTION}

There is evidence that percutaneous coronary intervention (PCI) in patients with stable coronary artery disease (CAD) offers no survival benefit over pharmacological therapy. Furthermore, benefits regarding angina relief are similar to medical therapy. ${ }^{12}$ However, various studies show that patients

\section{Strengths and limitations of this study}

- Until today, there has been limited research on patient factors; our study offers a new perspective on the overuse of cardiac catheterisation

- Based on 25 interviews, we identified six factors that influence asymptomatic patients' decision making regarding repetitive coronary angiographies.

- The preselection of patients by physicians may have caused a bias.

- The study was conducted in a single geographical region; it may not be representative for other healthcare systems.

- Overall, patients trusted their physicians and voiced little doubt on their recommendations.

and physicians agree to pursue elective cardiac catheterisation (CC) and possible $\mathrm{PCI}^{3}$ in stable CAD.

In 2014, a US national survey with 600 physicians showed that nearly $50 \%$ of physicians were asked for an unnecessary test or procedure by at least one patient per week. Thirty per cent of physicians even reported that this happened more than just once a week. ${ }^{4}$ Overuse of CC amounts to $4 \%-18 \%$ in the $\mathrm{USA}^{5}$ and is also reported for Germany in comparison with neighbouring European Union countries. ${ }^{6}$ Overuse of CG is not only risky for patients in the short term but is potentially associated with avoidable radiation exposure. A German study on multiple procedures and cumulative individual radiation exposure in interventional cardiology calculated exposures of up to $185 \mathrm{mSV}$ in individual patients. ${ }^{7}$ Also, it is assumed that at least US $\$ 158$ billion are spent on unnecessary treatments, ${ }^{8}$ that is, $30 \%$ of healthcare expenditures in the USA are preventable. ${ }^{9}$ Physicians have been reported to follow patients' requests for unnecessary coronary procedures ${ }^{10}$ despite guidelines that recommend a stepwise diagnostic approach with 
non-invasive testing prior to $\mathrm{CG}$ for stable coronary disease. ${ }^{5}$ While the role of healthcare systems and physicians has been widely studied, ${ }^{5}$ little is known on how patients contribute to decisions on unnecessary invasive procedures.

This qualitative study of patients with $\mathrm{CAD}$ from German teaching practices aims at identifying patient factors which contribute to and/or prevent the overuse of $\mathrm{CC}$ in stable CAD.

\section{METHODS AND ANALYSIS}

As detailed in our methods paper, ${ }^{11}$ we conducted patient interviews in two German teaching practices affiliated with our Institute for General Medicine. Teaching physicians asked patients with a history of acute coronary syndrome (ACS) or known CAD who had undergone at least one or, better yet, multiple CC procedures with or without an intervention, for voluntary participation in the study. Patients with known anxiety disorders were excluded to avoid a response bias regarding fears and anxieties. Two patients refused to participate because of lack of time; 25 patients agreed and gave written consent. The interviews were arranged by the practice: contact details were made available to the interviewer who then contacted the patients by phone. The interviewer was a female researcher (AH) with a sociology background who is qualified and experienced in qualitative research methods and interview techniques. The interviews were conducted in a treatment or meeting room in the practices with only the participant and the interviewer present. The interviewer introduced herself as a non-medical member of the research team from the institute and a member of the study project. After conducting 25 interviews, the so-called 'saturation point' was reached. Methodologically, this means that no new contents regarding the research question was recorded during the last interviews. No repeat interviews were conducted. All patients were given the opportunity to review the interview transcripts, but no participant took advantage of this.

\section{Patient and public involvement}

The research question was triggered by observations of German general practitioners who reported difficult encounters with asymptomatic patients with CAD and their demands for invasive rather than non-invasive testing. To better include patients' views in the interview guide, the interviewing researcher attended a coronary catheterisation laboratory for 1 day where she was given the opportunity to talk to patients before and after their procedures. Beyond this, patients were not involved in the development of the research question or the study design, but were recruited as participants. During the interviews, patients actively took part in the study. Patient representatives will be informed about the study results and asked to support dissemination.

\section{Analysis}

The analysis was carried out in different stages following the methodological approach of a content analysis by
Mayring $^{12}$ : the interviews were recorded and transcribed in full. Following anonymisation, the language material was reduced by determining evaluation units and by categorising subject areas. Each subject area represented a substantial meaning of the statements made by the patients at a higher abstraction level. The meaning of the statements, but not the frequency of occurrences, was a criterion for the categorisation. Each evaluation unit was encoded by two independent evaluators: the interviewer $(\mathrm{AH})$ and a general physician affiliated with the research team of the institute (DD). The computer program atlas. ti (V.7 and V.8) was used for the analysis. The results of the analysis by the two evaluators were compared with one another and were then used to improve the categorisation systems and coding guides. The final version is detailed in online supplementary appendix 1 . After that, the first evaluator reanalysed the entire material using the final version of the categorisation. A 10\% random sample of the coded material was reviewed by the second evaluator to ensure coding quality (see online supplementary appendix 1 for details). To further differentiate factors fostering CC within the specifics of the German healthcare system, we reanalysed quotations of aspect 2 (CC intervention) after scientific reviewer comments.

\section{RESULTS}

Twenty-four interviews with 25 patients were conducted. One interview was conducted with a couple who wished to be interviewed together as both were suffering from CAD. Seventeen $(68 \%)$ patients were male, the average age was 73.9 years (range 53-88 years). In total, 16:24:48 hours of interview material was collected with an average duration of 41:02 min per interview. The shortest interview lasted 18:12 min, the longest 01:18:10 hours. All patients suffered from CAD and had undergone at least one CC. Further details on the patients cited in this article are provided in online supplementary appendix 2 .

Our first categorisation revealed four factors that influence decision making regarding CC: (1) physicianpatient relationship, (2) patient characteristics (fear of another cardiac event), (3) patient information (disinterest in and lack of disease-specific knowledge) and (4) issues relative to CC in Germany. The reanalysis of the fourth factor revealed the three aspects: unquestioned acceptance of prescheduled appointments, helplessness in situations with varying opinions on the required level of care, patients' experiences that repeated interventions do not result in a change of health status or medical care. Thus, our final analysis comprised the following six factors that contribute to and/or prevent overuse of CC from a patient's perspective:

1. Unquestioned acceptance of prescheduled appointments for procedures and convenience

2. Helplessness in situations with varying opinions on the required level of care

3. Disinterest in and lack of disease-specific knowledge

4. Fear of another cardiac event 
5. Relationship between patient and (primary care) physician and

6. Patients' experiences that repeated interventions do not result in a change of health status or medical care.

As outlined in the subsequent citations, several of these factors typically played a role for each patient. Also, given the complexity of the topic, these aspects may occur simultaneously.

\section{Unquestioned acceptance of prescheduled appointments for procedures/convenience}

Patients reported follow-up appointments for the next CC which were already scheduled and communicated in the medical report after their last CC. This is an interesting observation, as it shows the interplay of a healthcare system factor (hospitals schedule these appointments automatically) and patients' uncritical acceptance. However, many patients were glad to accept the appointments without questioning the need for another CC, as it meant that they did not have to arrange an appointment themselves:

I can't possibly list all of them [the CCs]. Nine, maybe I've had nine or ten, and another one for my leg. [...] Those were all carried out as check-ups and not because something happened [...]. I always asked for a copy of my medical report at the hospital. [...] Two or threedays before the appointment I would call them and ask: 'Is the appointment still scheduled?' and they would say 'Yes, it is'. And then I said: 'Then I will be there.' I always went to the appointments scheduled in the medical report. [...] I never went because of actual complaints. (patient_6)

It appears that patient_6 wanted to be a 'model patient' and wanted to keep the appointment given to him by accepting his physician's advice unquestioned. The fact that he went to the follow-up appointment without actually having any complaints shows that there is a misconception about the need for CC. Below, patient_2 decided for himself that he really needed the appointment, not based on a medical requirement but rather on his own beliefs, although some subtle doubt remained:

When I got the medical report, the new appointment was already in it. They just scheduled it. (Reads out loud.) 'Presuming you agree, we have scheduled an appointment for $18 / 03 / 2005$.' That was the report from the 8th of April and then I was directly//Anew appointment was//Well, basically I was fine with all that, because it meant that I did not need to make an appointment myself. I kept telling myself that the appointment was essential and that I should just have it. (patient_2)

Similar to patient_2, another patient said that agreeing to have an intervention without knowing if there was really a need for it was as a matter of convenience, because it would have taken him some time to get an appointment with a cardiologist:
He [the physician] said that it would be better to have a closer look at it [an abnormal ECG]. If I go to the cardiologist, I have to wait a year before I can get an appointment. [...] So I said [...]: I'll just have another cardiac catheterization, because, let's be honest, it's much faster. I got an appointment within 14 days and then I had the procedure. (patient_23)

\section{Helplessness in situations with varying opinions on the required level of care}

Patients reported differences in recommendations between hospitals and primary care physicians. One patient described experiencing a conflict between trusting the professional competence of his primary care physician and the hospital physicians, who encouraged him to undergo recatheterisation for follow-up purposes. The patient appeared to be well informed about CC, talked about risks that are associated with CC and said that non-invasive procedures may have been just as effective in diagnosing anomalies. The following quote demonstrates his helplessness about whose advice to follow and how to make the right decision:

Half a year later they sent me an invitation, in fact they had already told me before that I should go there again to have another cardiac catheterization just to make sure that everything was okay. I then talked to my primary care physician and she advised me against having another CC, because those kinds of anomalies can also be discovered in simple examinations like a stress ECG. And because cardiac catheterization is associated with certain risks. They also told me that in hospital, but I was unsure and decided to follow the hospital's recommendation, because they said that they carried out the examination and would recommend having another catheterization based on the findings. I had a slightly better relationship to the hospital, because I had no idea about the subject matter and they had helped me in the past. Knowing what I know now, I may have decided differently, because I now visit my primary care physician for regular check-ups [...]. Interviewer: Does that mean you had the cardiac catheterization in hospital [...]. What did they find? (3): They didn't find anything. Everything was fine. (patient_3)

\section{Disinterest in and lack of disease-specific knowledge}

Some patients did not ask questions about their health because of a lack of interest in the disease and treatment, as illustrated by patient_11, who had deliberately decided not to learn about his disease:

Interviewer: Do you try to get information on your disease, do you read up on it? Patient: Absolutely not! No! I don't want to hear about illnesses. I just don't want to know about that stuff. (patient_11)

Furthermore, patients showed their disinterest by not dealing with the details of the invasive procedure and by 
reacting indifferently and impassively to the intervention. This refusal to accept the role of a competent patient who acts as an informed decision-maker is also reflected by a lack of knowledge of the medical terminology used:

Patient: That stinnet or stint or whatever it is called. Interviewer: You mean a stent? Patient: I don't remember things like that. I just don't care! (patient_14)

Patients often reported that they did not ask about the treatments and did not question the decision for an invasive procedure, because

The other question is whether I understood everything. [...] If they [the physicians] believe that it [a CC] has to be done, then they must be right. Questions only ever came up later. (patient_8)

Once again, it is obvious that patients accepted their physicians' advice unquestioned and were well aware of the fact that they did not fully understand the situation. This combination of factors, that is, patients' lack of information and healthcare system-driven physician factors (insufficient information about guideline-recommended approaches and physicians' preferences towards interventional procedures), increases the risk of overuse. Patient_2 described his situation as a kind of a vicious cycle, thus emphasising the helplessness of his situation:

And soon enough I was going around in circles.

The patient eventually got stuck in a cycle of recurring interventions.

\section{Fear of another cardiac event}

A heart attack or chest pain was described as a lifechanging experience. Patients expressed different emotions regarding their disease and the treatments. The key emotion described was fear in general and fear of another cardiac event in particular. This fear can manifest in different ways: starting with slight uncertainty about whether they are making the right decision (this, in turn, is closely connected to lack of knowledge), and culminating in panic attacks and fear of death.

Some patients explained that their fears were driven by experiences of family members or friends. Others knew people who had suffered or even died of heart disease. These patients consciously decided in favour of undergoing CC in order to prevent a fatal outcome:

My father had a heart attack, my mother had liver cirrhosis. And my sister recently died of breast cancer. She never went in for a check-up. My other sister had a cyst in her breast. I always think about these things and tell myself: I'd better have the check-up, just to make sure. (patient_7)

Mainly elderly patients claimed they were afraid of dying soon and were therefore willing to have the examination to prevent possible 'risk factors':

I prefer to play it safe rather than wait for something to happen. I don't have that much time left.' (patient_6)
Other patients reported acute fears for one's life or panic attacks, particularly shortly after suffering an event:

I must say, I feel very well taken care of here. But I used to come here for any- and everything. You just feel scared. It's like having a panic attack. (patient_9)

This attitude develops from patients' fear of another cardiac event. A younger patient who suffered his first heart attack in his mid-40s described feeling vulnerable because he was afraid of being a high-risk patient and he felt the need to act in order to prevent another potentially life-threatening event:

In the past I sometimes came back here [to the primary care practice] in the afternoon to have an ECG simply because I thought it was starting up again. You get so worked up about it. The body seems to respond quite strongly to what is going on in the head. But then [after the ECG] I felt reassured because they would tell me that everything was in order. Strangely enough, the feeling was gone after that. (patient_22)

This understandable insecurity was cited as one of the factors that drive the high number of doctor's visits and unnecessary CCs. Patients claimed that they decided in favour of undergoing CC to alleviate their fears and be reassured that their heart is healthy:

I once convinced myself that I should have another examination, because I had pain here, only here, and only while I was doing exercise, for example when I walked up a hill. I thought there could be no harm in having another examination. But they didn't find anything. (patient_8)

\section{Relationship between patient and (primary care) physician}

The relationship between the patient and his physician played a major role in decision making about care processes. Our patient interviews showed that the patients' trust in their physicians both drove and prevented overuse. Patients reported that they did not feel the need to ask questions because of their strong trust in the physicians and the belief that the physicians was only trying to help them; in other words, patients did not believe that physicians had any motive other than curing them:

Above all, that's what I believe: They just want to help. And the procedure is meant to help. It wouldn't hurt to be a bit more positive. They wouldn't just put you through such an ordeal without good reason. They just want to have a look. Thank God this procedure exists. (patient_4)

Strong trust in physicians also contributed to a factor that can prevent the overuse of CC. Patients described their relationship to their primary care physician as being one of confidence:

I am in the best possible hands. (patient_4); 
I do anything she [the primary care physician] says. (patient_9).

When asked whether he searched for information about this disease independently, one patient answered:

You always hear and read and see things. Basically, I always rely on what the physicians say. It remains to be seen whether they were right. They have already made mistakes in the past. (patient_1)

\section{Patients' experiences that repeat interventions do not result in a change of health status or medical care}

One patient who had repeatedly undergone 6 monthly invasive angiographies reported his change of heart over time. He reflected that he would have decided differently with his current knowledge, and that his trust in his primary care physician had increased over the years:

Eight years later I can imagine that, with all those six-monthly examinations with stress ECGs, you would be sure to notice pain or other complaints. (patient_3)

Another patient realised that merely following prescheduled appointments for procedures did not help him and did not improve his health status. He learnt that a cardiac event would not go unnoticed and that an appropriate intervention would be found when the time was right:

I had just had surgery in 2009 and I said to myself: Now I have some peace. And then I thought: In the last 14 years you have only had these [coronary] repairs. I was literally waiting for the doctor to tell me that I have to have another repair. But they do this only if you actually have severe discomfort. That is what I read between the lines when I once asked primary care physician: Look, shouldn't I be having another cardiac catheterization? No, you don't have any symptoms. Apparently, I would notice when something is about to happen. (patient_2)

Also, one patient reported that he had had two consecutive CCs, but the physicians found no relevant outcomes. He then decided that he would never undergo such an intervention again:

Interviewer: That means you underwent catheterization twice? patient_12: Two consecutive catheterizations, because apparently they discovered something, but then during the second intervention they found nothing. And that's when I decided that I would never agree to do this again. (patient_12)

\section{DISCUSSION}

Our analysis identified six patient factors which foster the overuse of CC: (1) unquestioned acceptance of prescheduled appointments for procedures/convenience; (2) disinterest in and/or lack of disease-specific knowledge;
(3) helplessness in situations with varying opinions about the care needed; (4) fear of another cardiac event, (5) patient-physician relationship and (6) the patient's experience that repeat interventions do not result in a change of health status or care.

Some of our findings are in line with the results of a systematic review of 17 studies by Ager $e t$ al which addressed patient-reported factors influencing the decision for contralateral prophylactic mastectomy (CPM) in women with early breast cancer. We chose this comparison because the patients' situations are similar in terms of the intervention strategy offering no survival benefit. ${ }^{13}$ Also, both breast cancer and heart disease are associated with a risk of recurrence and even death. In agreement with Ager $e t a l,{ }^{14}$ we found that fear and the patient-physician relationship were crucial factors. Against the backdrop of the fear of disease recurrence, patients with both clinical pictures believed that additional interventions would allay their concerns and even lower their personal risk. In another study of CPM patients, Greener coined this the 'significance of patients' being proactive' about the treatment ${ }^{15}$; to a certain degree, this is similar to the unquestioned acceptance of prescheduled appointments in our study. Patients with CAD reported feeling good and worrying less about their disease if they stuck to the recommended appointments.

The physicians' advice had a high impact in both patient groups. In addition, patients undergoing CPM reported that they had been influenced by their spouses or the family; in our interviews, however, this did not play a role. Only few patients with repeat CCs believed them to be inappropriate for their health status and described a change of heart during the course of their disease: while they initially followed the cardiologists' recommendation, they later trusted their general practitioners' advice that no further invasive testing was needed. Interestingly, the issue of overuse and the lack of a survival benefit were addressed more indirectly: patients reported that they abstained from further interventions after realising that the repeat procedures changed neither their health status nor their medical care, yet they did not reflect on potential driving forces on behalf of the cardiologists or the healthcare system. This is interesting, as our study was conducted in a healthcare system with known overuse of CC driven by regional reimbursement strategies. ${ }^{16}$ Our patients with CAD did not voice any of the motives that were identified in a qualitative study among 20 Californian cardiologists in 2007: despite evidence that PCI does not provide a benefit for patients with stable $\mathrm{CAD}$, cardiologists believed in the benefits of treating ischaemia, especially with drug-eluting stents. Cardiologists were afraid of regretting their decision not to perform the invasive procedure if a patient died from CAD later on. Also, cardiologists wanted to grant their patients' requests for another CC to alleviate patients' anxieties. ${ }^{10}$ A 2014 National Survey of Physicians among 600 practising US physicians confirmed that unnecessary tests and procedures are ordered merely for the physicians' reassurance: 
reasons included concerns about malpractice issues $(52 \%)$, just to be safe $(36 \%)$ and wanting more information to gain certainty (30\%). Patients' insistence (28\%), the desire to keep patients happy (23\%) and a lack of time for the patient (13\%) also contributed to this development. ${ }^{4}$ Together with the importance of patients' fears regarding making a decision in favour of (repeat) interventions, these findings are of utmost important, as they suggest a synergy of physician and patient fears/uncertainties as driving factors for overuse.

Focusing on patients' lack of knowledge and helplessness, a Dutch study in 201 patients with CAD showed that the prevalence of inadequate health literacy is high in patients with CAD: $5 \%(\mathrm{n}=11)$ had difficulties understanding and applying health information, $18 \%(\mathrm{n}=34)$ had inadequate reading skills and 52\% ( $\mathrm{n}=103)$ had difficulties understanding and applying written information. This was related to cardiovascular outcomes and secondary prevention. ${ }^{17}$ The need for better patient education is supported by our study: patients self-reported their lack of disease-specific knowledge and the difference in knowledge and experience between themselves and the specialist. Because even very well-educated patients need to trust their physicians' professionalism, reasonable measures to decrease overuse include better healthcare system regulations, professional education to promote evidence-based care as well as strategies for better patient participation in decision making. In recent years, there has been a cultural shift towards shared decision making, ${ }^{18} 19$ which means that clinicians and patients make informed decisions together to support patients' involvement and autonomy ${ }^{20}$ instead of clinicians merely making decisions on behalf of patients. Screening and diagnostic tests in particular are considered suitable for shared decision making. ${ }^{21}$ Our study shows that various anxieties and misconceptions about coronary disease foster patients' consent to unnecessary coronary procedures. Thus, to better implement evidence-based shared decision making for coronary disease, future strategies must consider and address these emotional and educational aspects.

One strength of our study is that it addresses patient factors within the context of CC overuse. However, several study limitations need to be considered. First, the preselection of patients by the family physicians may have caused a bias, although we tried to minimise this bias by selecting a rather large number of patients from two practices with multiple physicians. Second, the study was conducted in only one region and the results may not necessarily be transferable to other healthcare systems. Third, interviews typically reflected patients' current thoughts, which may not necessarily be consistent with a change of heart that may occur during the course of their disease. Future research should address the interplay between physician and patient factors as contributors to overuse.

Acknowledgements We thank every participating patient.

Contributors AH: complemented the study concept, conducted and transcribed the interviews, analysed the interviews, wrote the first draft of the manuscript. DD: analysed the interviews. BW: developed the study idea and concept, revised the manuscript. All authors critically reviewed the first draft and provided feedback on it. All authors read and approved the final manuscript.

Funding The authors have not declared a specific grant for this research from any funding agency in the public, commercial or not-for-profit sectors.

Competing interests None declared.

Patient consent for publication Not required.

Ethics approval Ethics Committee of the Faculty of Medicine at the University of Duisburg-Essen, Germany (15-6448-B0).

Provenance and peer review Not commissioned; externally peer reviewed.

Data sharing statement Data is available on request. Please contact the corresponding author.

Open access This is an open access article distributed in accordance with the Creative Commons Attribution Non Commercial (CC BY-NC 4.0) license, which permits others to distribute, remix, adapt, build upon this work non-commercially, and license their derivative works on different terms, provided the original work is properly cited, appropriate credit is given, any changes made indicated, and the use is non-commercial. See: http://creativecommons.org/licenses/by-nc/4.0/.

\section{REFERENCES}

1. Jeremias A, Kaul S, Rosengart TK, et al. The impact of revascularization on mortality in patients with nonacute coronary artery disease. Am J Med 2009;122:152-61.

2. Boden WE, O'Rourke RA, Teo KK, et al. Optimal medical therapy with or without PCl for stable coronary disease. N Engl J Med 2007;356:1503-16.

3. Rothberg MB, Sivalingam SK, Ashraf J, et al. Patients' and cardiologists' perceptions of the benefits of percutaneous coronary intervention for stable coronary disease. Ann Intern Med 2010;153:307-13.

4. PerryUndem Research/Communication. Unnecessary Tests and Procedures. The health care system: what physicians say about the problem, the causes, and the solutions. results from a national survey of physicians: PerryUndem Research/Communication, 2014.

5. Scanlon PJ, Faxon DP, Audet AM, et al. ACC/AHA guidelines for coronary angiography: executive summary and recommendations. A report of the American College of Cardiology/American Heart association task force on practice guidelines (Committee on Coronary Angiography) developed in collaboration with the society for cardiac angiography and interventions. Circulation 1999;99:2345-57.

6. Nichols M, Townsend N, Scarborough P, et al. European cardiovascular disease statistics, 2012nd edn. Brussels, Sophia Antipolis: European Heart Network; European Society of Cardiology, 2012.

7. Weltermann BM, Rock T, Brix G, et al. Multiple procedures and cumulative individual radiation exposure in interventional cardiology: a long-term retrospective study. Eur Radiol 2015;25:2567-74.

8. Sutkowi-Hemstreet A, Vu M, Harris R, et al. Adult patients' perspectives on the benefits and harms of overused screening tests: a qualitative study. $J$ Gen Intern Med 2015;30:1618-26.

9. Berwick DM, Hackbarth AD. Eliminating waste in US health care. JAMA 2012;307:1513-6.

10. Lin GA, Dudley RA, Redberg RF. Cardiologists' use of percutaneous coronary interventions for stable coronary artery disease. Arch Intern Med 2007;167:1604-9.

11. Herwig A, Weltermann B. Study protocol for a matter of heart: a qualitative study of patient factors driving overuse of cardiac catheterisation. BMJ Open 2017;7:e017629.

12. Mayring P. Qualitative inhaltsanalyse: grundlagen und techniken. s.l: Beltz Verlagsgruppe, 2010.

13. Giuliano AE, Boolbol S, Degnim A, et al. Society of Surgical Oncology: position statement on prophylactic mastectomy. approved by the society of surgical oncology executive council, March 2007. Ann Surg Oncol 2007;14:2425-7.

14. Ager B, Butow P, Jansen J, et al. Contralateral prophylactic mastectomy (CPM): a systematic review of patient reported factors and psychological predictors influencing choice and satisfaction. Breast 2016;28:107-20.

15. Greener JR, Bass SB, Lepore SJ. Contralateral prophylactic mastectomy: a qualitative approach to exploring the decision making process. J Psychosoc Oncol 2018;36:145-58.

16. Flachskampf FA, von Erffa J, Seligmann C. Reimbursement and the practice of cardiology. J Am Coll Cardiol 2012;59:1561-5. 
17. van Schaik TM, Jørstad HT, Twickler TB, et al. Cardiovascular disease risk and secondary prevention of cardiovascular disease among patients with low health literacy. Neth Heart $J$ 2017;25:446-54.

18. Stiggelbout AM, Van der Weijden T, De Wit MP, et al. Shared decision making: really putting patients at the centre of healthcare. $B M J$ 2012;344:e256.
19. Coulter A. Shared decision-making: the debate continues. Health Expect 2005;8:95-6.

20. Elwyn G, Laitner S, Coulter A, et al. Implementing shared decision making in the NHS. BMJ 2010;341:c5146.

21. Barry MJ, Edgman-Levitan S. Shared decision making - The pinnacle of patient-centered care. N Engl J Med Overseas Ed 2012;366:780-1. 